\title{
Dielectric-Loaded Conformal Microstrip Antennas for Versatile In-Body Applications
}

\author{
Denys Nikolayev, Member, IEEE, Wout Joseph, Senior Member, IEEE, Anja Skrivervik, \\ Maxim Zhadobov, Senior Member, IEEE, Luc Martens, Member, IEEE, and Ronan Sauleau, Fellow, IEEE
}

\begin{abstract}
Wireless implantable bioelectronics and accurate in vivo characterization of path loss require efficient and robust in-body antennas. Loading electric antennas with the effective permittivities higher than that of surrounding tissues is a promising solution. In this study, proof-of-concept conformal microstrip antennas are proposed. The antennas are optimized for a standard impedance of $50 \Omega$ and operate in all high-watercontents tissues. Integral ground plane shields the antenna from inner circuitry. The radiation efficiencies are $0.4 \%, 2.2 \%$, and $1.2 \%$ for the $(434,868$, and 1400$)-\mathrm{MHz}$ designs, respectively, when computed in a $\varnothing 100-\mathrm{mm}$ spherical phantom with muscleequivalent properties. The efficiencies are compared to the fundamental limitations and closely approaches them. Specific absorption rates are evaluated, and the corresponding maximum input power levels are established. Prototypes are fabricated and characterized to validate the design.
\end{abstract}

Index Terms-biomedical telemetry, capsule antenna, conformal antenna, implantable, in-body, ingestible, microstrip antenna, robustness.

\section{INTRODUCTION}

W IRELESS implantable bioelectronics is a fast growing field having many potential applications in medicine, clinical research, professional sports, occupational health, and defense [1]-[3]. In-body devices require antennas to communicate with external equipment. Establishing a robust link between an in-body device and external equipment is a major challenge because of low radiation efficiencies $(\eta \sim 0.1 \%)$ due to high power dissipation in tissues [4], [5] and miniature size of the radiating elements. Moreover, efficient inbody antennas allow for minimization of measurement errors in path loss characterization in vivo [6]-[8], especially for deep-body applications or in large animals. In this frame, a wide range of antennas has been recently reported for bodyimplantable capsule applications, e.g. [9]-[17]. Robustness to heterogeneous and uncertain electromagnetic (EM) properties

Manuscript received September 13, 2019; revised October 18, 2019.

This work was supported by the European Union through the European Regional Development Fund (ERDF), and by the French region of Brittany, Ministry of Higher Education and Research, Rennes Métropole and Conseil Départemental 35, through the CPER Project SOPHIE / STIC \& Ondes. (Corresponding author: Denys Nikolayev.)

D. Nikolayev and A. Skrivervik are with the Microwave and Antenna Group (MAG), École Polytechnique Fédérale de Lausanne (ÉPFL), CH-1015 Lausanne, Switzerland (e-mail: d@deniq.com).

W. Joseph and L. Martens are with the imec / Ghent University, BE-9052 Gent, Belgium.

M. Zhadobov and R. Sauleau are with the Univ Rennes, CNRS, Institut d'Électronique et de Télécommunications de Rennes, UMR-6164, FR-35000 Rennes, France. of tissues was considered in [18]-[21], and a reconfigurable capsule-conformal antenna without nulls in its radiation pattern was proposed in [22]. Effects of dielectric loading on radiation performance were studied in [23]-[25]. Although high-permittivity ceramics, such as alumina $\left(\varepsilon_{r} \approx 10\right)$ or zirconia $\left(\varepsilon_{r} \approx 29\right)$, have been applied for miniaturization of inbody antenna superstrates [12], [14], [20], no practical designs have been reported for materials having the higher permittivity than high-water-content tissues (i.e. $\varepsilon_{r}>50$ ). Such materials would allow for taking advantage of loading by tissues while reducing losses in the near-field [26], [27].

This Letter proposes superstrate-loaded $\left(\varepsilon_{r} \approx 80\right)$ capsuleconformal antennas operating at 434,868 , and $1400 \mathrm{MHz}$. The choice of frequencies is motivated by prior studies on the optimal frequencies for in-body applications [28]. The microstrip designs feature an integral ground plane shielding the antenna from the capsule interior. Therefore, arbitrary configurations of capsule inner components are possible. The antennas are well matched to $50 \Omega$ and operate in all high-water-contents tissues $\left(\left|S_{11}\right|<-10 \mathrm{~dB}\right)$. The proposed antenna structure induces relatively low specific absorption rates (SAR) that allow for the maximum input powers of about $16 \mathrm{dBm}$. The antenna design remains simple and conforms to the inner wall of the capsule shell, therefore, occupying a small volume. Operating principle of the antenna is discussed, and prototypes are fabricated to validate the design.

\section{Antenna Design}

1) Geometry and Materials: As Fig. 1a shows, the proposed designs fit into $29 \times \varnothing 9 \mathrm{~mm}$ capsule. The shell is made of biocompatible low-loss ceramic with $\varepsilon_{r} \approx 80$ close to the maximum value encountered in a human body [29]. Such EM properties are characteristic to perovskite materials (barium/calcium titanates in our case). This loadingby-superstrate approach improves wave-impedance matching between the antennas and surrounding tissues [24] and significantly enhances the achievable radiation performance [23]. In view of the limitations on machining precision of ceramics, we used a 1-mm-thick capsule shell. Note that decreasing the shell thickness results in a wider bandwidth but reduces the radiation efficiency. Non-biocompatible electronics and circuitry are sealed inside of the biocompatible and nonbiodegradable insulation as in [30]-[32]. For further safety, the capsule can be filled with biocompatible epoxy as in [19]. The antennas are designed to operate on a flexible 101.6- 
TABLE I

Dimensions of the Proposed ANTENNAS (Units: MiLLIMETERS)

\begin{tabular}{rlccll}
\hline$f_{d}$ (MHz) & $l_{1}$ & $l_{c}$ & $w_{1}$ & $w_{2}$ & $w_{c}$ \\
\hline 434 & 2.5 & 0.67 & 11.6 & 0.18 & 0.9 \\
868 & 3.34 & 0.95 & 6.71 & 0.3 & 0.3 \\
1400 & 1.9 & 0.47 & 4 & 0.5 & 0.3 \\
\hline
\end{tabular}

$\mu \mathrm{m}$-thick (4 mil) Rogers 3850HT substrate $\left(\varepsilon_{r}=2.9\right.$ and $\tan \delta=0.002$ ). Low substrate-to-superstrate permittivity ratio results in a higher antenna impedance (this helps achieving $50-\Omega$ matching) and contributes to the radiation resistance [33]. On the other hand, increasing the substrate permittivity enhances the antenna robustness since it would diminish $\mathbf{E}$ field fringing into tissues. Fig. 1b shows the geometry of the antennas, and Table I lists the dimensions.

Inserting a flexible substrate into the shell would inevitably create a thin air gap in-between. This gap affects the effective permittivity of the antenna medium. In addition to weakened dielectric loading, this impairs the reproducibility since it is difficult to control the gap. One of the ways to address this is to fill the gap with pure deionized water. Having its permittivity close to the shell $\left(\varepsilon_{r} \approx 80\right)$, water provides a smooth waveimpedance transition between the surrounding tissues and the antenna (Fig. 1c). Water below the ground plane contributes insignificantly to the dielectric loading: as Fig. 2 shows, almost no E-field penetrates into the capsule interior. Since $\varepsilon_{r}$ of water is temperature-dependent, we verified its effect on the antenna in the range of $32-42{ }^{\circ} \mathrm{C}$. First order Debye model was used for the validation; the results show no significant effect of the temperature $\left(\delta\left|S_{11}\right|<1 \%\right)$. Note that pure water is used here as a technical proof of concept. For industrial applications of the proposed designs, a more practical solution would be embedding the antenna into the superstrate using ceramic-powder charged polymers or epoxies [34], [35].

2) Operating Principle and Miniaturization: The proposed antennas are of a cylinder-conformal microstrip type and are designed to operate similarly to a half-wave patch. The radiating edges are spaced out between two extremities of the cylinder, and such a configuration allows for the improved aperture efficiency. Fig. 2 shows the electric field distribution for the final design: one can observe the antiparallel $\mathbf{E}$-field maxima generated at the cylinder extremities and the zero $\mathbf{E}$ field in the middle of the antenna. The distribution of the surface current density corresponds to a typical patch with the maximum in the middle and the minima at the edges.

Fitting a $\lambda / 2$ antenna to a 29-mm-long capsule requires miniaturization at all design frequencies $f_{d}=$ $(434,868,1400) \mathrm{MHz}$. The miniaturization is partly achieved via dielectric loading by surrounding tissues and the superstrate. A modified H-antenna technique [36] was used to further miniaturize the antenna. The resulting design consists of two identical radiating elements connected by a highimpedance microstrip line (Fig. 1b). The latter also contributes the impedance matching by increasing $\Re\left(Z_{A}\right)$ since a conventional thin-substrate patch antenna would have too low radiation resistance in the high-permittivity medium [37, Ch. 14]. The stepped transition of the radiating elements into the

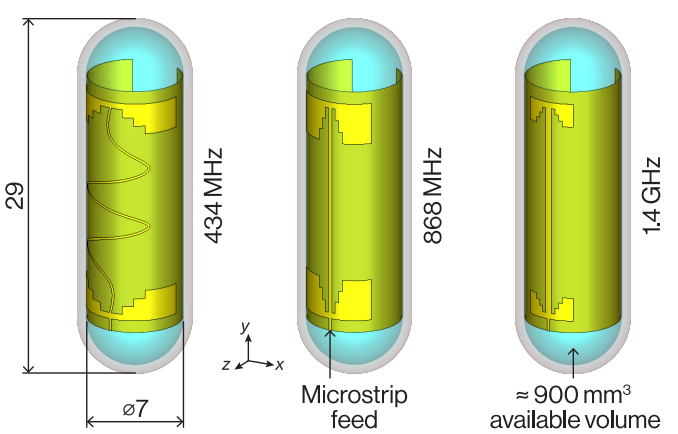

(a)

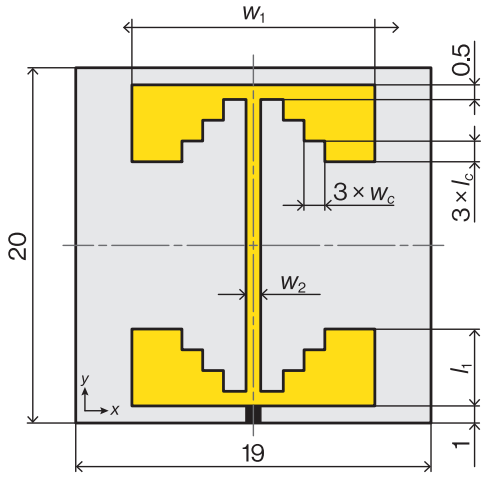

(b)

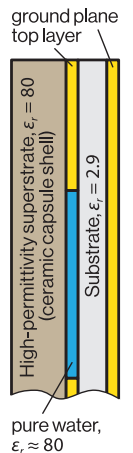

(c)
Fig. 1. (a) Proposed capsule-conformal microstrip antenna designs and dimensions (units: millimeters). (b) Planar geometry and dimensions (not to scale). (c) Antenna stack-up and its dielectric properties. Pure water provides waveimpedance matching with the superstrate for consistent dielectric loading.

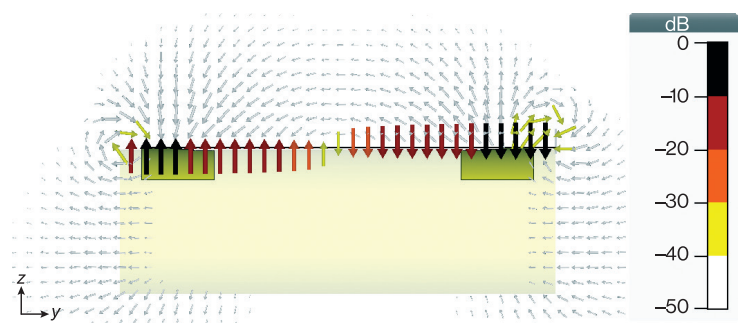

Fig. 2. Electric field generated by the proposed antennas corresponds to a conventional patch. This figure shows an $\mathbf{E}$-field distribution example in $x=0$ plane of the $868-\mathrm{MHz}$ design.

high-impedance microstrip increases the ratio of $w_{1}$ to the characteristic impedance [38] further increasing the electrical size of the antenna. To miniaturize the $434-\mathrm{MHz}$ design, the high-impedance microstrip was meandered and defined as a cubic spline (Fig. 1a). The spline interpolates seven equidistant points that span $\Delta y=17 \mathrm{~mm}$. In the $x$-coordinates, these points were defined as $\left[0-w_{s} 2 w_{s}-2 w_{s} 2 w_{s}-w_{s} 0\right]$; the optimized value of $w_{s}=3.1 \mathrm{~mm}$.

3) Numerical Modeling: We used CST Microwave Studio 2018 to develop the antennas [39]. Detailed description of the numerical approach is given in [40], [41]. Intersubject body and organ morphologies can vary strongly, and, in general, the exact location of a capsule in living tissues varies with time. Therefore, analyzing the antenna radiation performance in an anatomical phantom is impractical. First, the obtained antenna patterns are difficult to decorrelate from the phantom geometry. Second, since the results depend strongly on the 


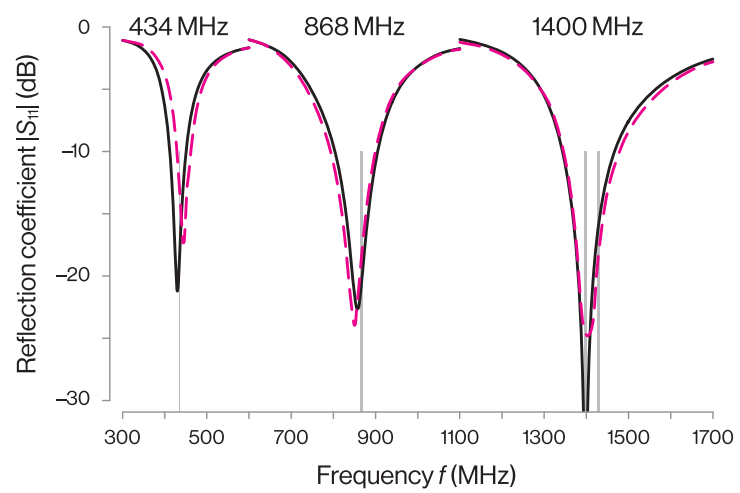

Fig. 3. Simulated (-) and measured (- - reflection coefficient $\left|S_{11}\right|$ of the antennas in a phantom with muscle-equivalent EM properties.

exact location and orientation of the antenna in a given tissue, it impairs the reproducibility of the results. For these reasons, it is important to establish a reference well-characterized phantom so that the results can be reproducible, and different designs can be gauged relatively to the maximum achievable efficiency in a reference phantom. Sphere is an obvious choice as this shape introduces isotropic, direction-independent losses conserving the intrinsic radiation pattern independently of the antenna orientation [25]. Moreover, the radiation performance in a simple phantom can be verified experimentally.

\section{Antenna Performance}

Fig. 3 shows the impedance characteristics of the proposed antennas in the muscle-equivalent environment. The $-10 \mathrm{~dB}$ bandwidths are $39 \mathrm{MHz}$ (fractional $\mathrm{BW}=9 \%$ ), $90 \mathrm{MHz}$ (10\%), and $124 \mathrm{MHz}(9 \%)$ for the $(434,868$, and 1400)-MHz designs, respectively. The obtained bandwidths fully cover the relevant bands of wireless communication standards such as ISM, MedRadio, LoRa, Sigifox, WMTS, etc.

To study the antenna impedance robustness (or its detuning immunity) to the variation of surrounding tissues, we varied the EM properties of the phantom ranging from the fatequivalent to the cerebrospinal-fluid-equivalent one. Owing to dielectric loading, the designs operate in all high-watercontents tissues (i.e. $\varepsilon_{r} \geq 40 \forall f_{d}$ [29]) with $\left|S_{11}\right|<-10 \mathrm{~dB}$. Note that the high-water-contents tissues include the gastrointestinal ones making the proposed antennas potentially suitable for ingestible applications. In fat, however, $\left|S_{11}\right| \approx-3 \mathrm{~dB}$ due to much lower $\varepsilon_{r}$ of fat compared to other soft tissues.

The integral ground plane on the bottom layer of the substrate (Fig. 1c) shields the antenna from the capsule interior making its performance independent of the device packaging. To confirm this, we simulated the capsule antennas filled with a PEC cylinder occupying the entire cylindrical volume beneath the antenna. The $\left|S_{11}\right|$ variation is insignificant $\left(\delta f_{\text {res }}<1 \%\right)$.

Fig. 4 depicts the radiation performance of the antennas. The maximum gains $G$ are $(-28.0,-16.0$, and -16.1$) \mathrm{dBi}$, and the radiation efficiencies $\eta$ are $0.4 \%, 2.2 \%$, and $1.2 \%$ for the (434, 868, and 1400)-MHz designs, respectively. Reduced $\eta$ at $1.4 \mathrm{GHz}$ (compared to $868 \mathrm{MHz}$ ) is due to the increased power dissipation in tissues. The $434-\mathrm{MHz}$ and $868-\mathrm{MHz}$ designs are omnidirectional with a typical dipole-like pattern of an
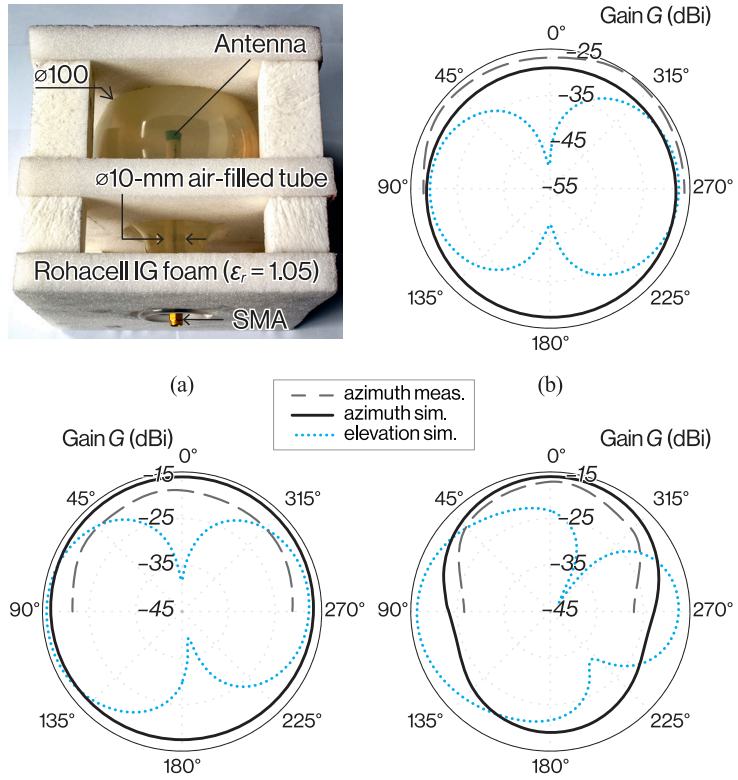

(c)

(d)

Fig. 4. Simulated and measured radiation performance of the antennas in $\varnothing 100$-mm spherical phantoms with muscle-equivalent properties. (a) The spherical phantom used for radiation measurements. SMA connects the AuT to an analog fiber-optic convertor (enprobe LFA-3). (b)-(d) Measured and computed radiation patters of (b) $434 \mathrm{MHz}$, (c) $868 \mathrm{MHz}$, and (d) $1400 \mathrm{MHz}$ designs. $0^{\circ}$-bearing aligns with the $z$-axis for the azimuth-plane pattern and with the $y$-axis for the elevation plane (axes defined on Fig. 1).

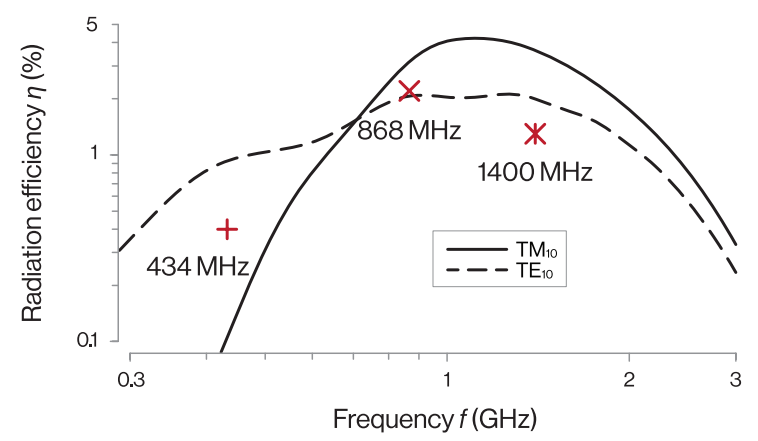

Fig. 5. Comparison of the proposed designs (red crosses) with the maximal achievable efficiencies of optimal $\mathrm{TM}_{10}$ and $\mathrm{TE}_{10}$ sources in a $\varnothing 100-\mathrm{mm}$ spherical muscle-equivalent phantom. The efficiencies of the optimal sources have been calculated using the methodology given in [25] for the following source parameters (units: millimeters): $L=18, R=3.5$, and $T=1$.

electrically small antenna. In contrast, the $1.4-\mathrm{GHz}$ design has the directivity $D=4 \mathrm{dBi}$. Using a single antenna of this type for ingestible capsule applications is thus suboptimal. However, a cylindrical array of such antennas makes it possible to realize beam-steering capabilities.

IEEE limits the maximum 1-g SAR to $1.6 \mathrm{~W} / \mathrm{kg}$ [42]. Simulated 1-g SAR $\left(P_{i n}=1 \mathrm{~W}\right.$, averaging according to IEEE C95.3) are $(34.16,34.40$, and 36.76$) \mathrm{W} / \mathrm{kg}$ for the $(434,868$, and 1400)-MHz designs, respectively. The proposed designs can therefore operate safely at the maximum input powers less than $(46.8,46.5$, and 43.5$) \mathrm{mW}$ or $(16.7,16.7$, and 16.4) $\mathrm{dBm}$. 


\section{A. Radiation Efficiency vs. Fundamental Limitations}

Radiation performance comparison with counterparts requires evaluating all antennas within the same phantom. Moreover, the maximum achievable efficiency of the antenna strongly depends on its size. The direct comparison, therefore, is inadequate. Instead, we propose gauging the design quality in terms of the obtained radiation efficiencies compared to the maximum achievable ones for a given antenna size in a standard phantom. The fundamental limitations on the radiation efficiency $\eta$ of finite-sized body-implanted capsules have been established in [25]. Fig. 5 benchmarks the proposed designs against the maximally achievable efficiencies of corresponding optimal $\mathrm{TM}_{10}$ and $\mathrm{TE}_{10}$ sources in a $\varnothing 100-\mathrm{mm}$ spherical muscle-equivalent phantom. The optimal sources have been calculated with the following parameters corresponding to the proposed designs: $L=18 \mathrm{~mm}, R=3.5 \mathrm{~mm}$, and $T=1 \mathrm{~mm}$.

Owing to $\varepsilon_{r}=80$ loading, the $434-\mathrm{MHz}$ design exceeds the $\eta$ of an unloaded $\mathrm{TM}_{10}$ source (i.e. loaded only by tissues). However higher $\eta$ can be achieved using a magnetic $\mathrm{TE}_{10}$ source. The $868 \mathrm{-MHz}$ antenna is the most efficient among the proposed. It overcomes $\max (\eta)$ of $\mathrm{TE}_{10}$ and closely approaches $\mathrm{TM}_{10}$. The radiation efficiency of the $1.4-\mathrm{GHz}$ design is below $\max (\eta)$ of both $\mathrm{TM}_{10}$ and $\mathrm{TE}_{10}$. Its increased directivity (Fig. 4d) implies existence of higher-order modes. It results in elevated power dissipation in the near field of the antenna [27, Fig. 6]. However, narrow $w_{1}$ makes it possible to form a circular array around the capsule.

\section{PROTOTYPING AND EXPERIMENTAL CHARACTERIZATION}

Laser ablation technique (LPKF ProtoLaser S) was used to pattern the antenna designs on the 101.6- $\mu$ m-thick (4 mil) Rogers ULTRALAM 3850HT substrate (Fig. 6a). The resulted antennas were inserted into a $28 \times \varnothing 9 \mathrm{~mm}$ ceramic shell (TCI Ceramics "K-80" [43], $\varepsilon_{r}=80 \forall f_{d}$ ) and sealed.

For the impedance and radiation characterization, we prepared liquid muscle-equivalent phantoms for each $f_{d}$. We used a water-sugar-salt formula [44] to achieve target EM properties. Pure deionized water was the bulk component of the phantom, sucrose $\left(\mathrm{C}_{12} \mathrm{H}_{22} \mathrm{O}_{11}\right)$ reduced the permittivity, and sodium chloride $(\mathrm{NaCl})$ increased the conductivity. EM properties were validated using SPEAG DAK-12 probe [45]. Fig. 3 demonstrates very good agreement in terms of the reflection coefficients $\left|S_{11}\right|$ of the fabricated antenna prototypes with the corresponding numerical results. The highest deviation of $\delta f_{d} \approx 7 \%$ for the $434-\mathrm{MHz}$ design could be attributed to relatively low tolerances of the laser-ablation method for patterning of the 0.18-mm-wide high-impedance transition (Fig. 6a). More precise manufacturing-such as photolithography-could further improve the agreement.

To characterize the radiation performance, we used a previously proposed approach [14]. The antenna under test (AuT) is centered inside of a $\varnothing 100$-mm spherical glass jar containing a muscle-equivalent phantom (Fig. 4a). An analog electrooptical convertor [46] feeds the AuT via an optical fiber that replaces the RF cable inside of an anechoic chamber and thus minimizes the signal distortion. AuT is located at the distance

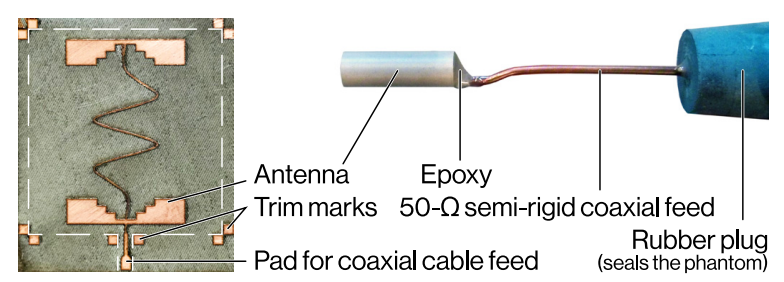

(a)

(b)

Fig. 6. Prototype manufacturing. (a) 434-MHz design patterned on a Rogers 3850 HT substrate. (b) The antenna soldered to a coaxial feed, rolled, and inserted into a ceramic shell. Araldite 2012 epoxy seals the cable side. Prior to characterization, the capsule is filled with pure water and sealed with a rubber plug (not shown). For the radiation measurements, the coaxial feed is insulated from the phantom with an air-filled $\varnothing 10-\mathrm{mm}$ polyamide tube.

$d=3 \mathrm{~m}$ from the measurement antenna fulfilling the farfield criterion of $d>2 \lambda \forall f_{d}$. The radiation was measured in the azimuth plane for $\theta \in\left[90^{\circ}, 270^{\circ}\right]$ due to the positioner angle limitations (see [14]). To derive the antenna gain from the obtained data, the gain substitution technique was used. A reference antenna of a known gain [47] substituted the AuT, and the measured gain of the reference antenna was used to calibrate the results. Figs. $4 \mathrm{~b}-\mathrm{d}$ show the far-field characterization results. The maximum measured gains $G$ are $(-25,-18$, and -17$) \mathrm{dBi}$ for the $(434,868$, and 1400$)-\mathrm{MHz}$ designs, respectively. The radiation patterns and maximum gain values are consistent with the simulated ones.

\section{Discussion And Conclusion}

This study proposes a family of in-body capsule-conformal microstrip antennas. Dielectric loading by a capsule shell with its permittivity $\left(\varepsilon_{r} \approx 80\right)$ greater than that of surrounding tissues was applied for the first time. This approach is based on the theoretical findings [25] that predicted higher maximum achievable in-body radiation efficiencies of dielectrically-loaded electric (i.e. E-coupled) antennas than the magnetic ones. Note that magnetic in-body antennas still show better efficiency when electrically small (Fig. 5). Choosing a microstrip design among other E-coupled antennas improves robustness to the inner circuitry and adjacent tissues [14], [19], [20]. The proposed designs feature an integral ground plane without any aperture making it fully shielded from the capsule interior. This has a double benefit of improving the EM compatibility and making the antenna impedance independent of the inner capsule components (e.g. batteries, circuits, and sensors). The proposed designs, therefore, demonstrate the feasibility of robust and efficient dielectric-loaded in-body capsule-conformal antennas.

The H-antenna miniaturization approach used in this study has its limitations. Specifically, for a given capsule size, further reducing the operating frequency $(f \lesssim 400 \mathrm{MHz}$ ) would result in too low radiation resistance and therefore a difficulty to attain $\Re\left(Z_{A}\right)=50 \Omega$. On the other hand, higher-frequency designs, such as $2.45 \mathrm{GHz}$, do not require miniaturization as only the dielectric loading suffices in this case. Simple conformal patch-antenna designs could be therefore synthesized. The proposed designs (both in planar and conformal realizations) can also find their applications in other non-medical fields 
where establishing robust communication links is required with devices in high-permittivity and lossy environments.

\section{ACKNOWLEDGMENT}

The authors would like to thank J.-C. Le Cun for the laser ablation, L. Verloock and M. Van den Bossche for helping with the assembly of the prototypes

\section{REFERENCES}

[1] E. Katz, Implantable Bioelectronics. Weinheim, Germany: Wiley, 2014.

[2] A. Yakovlev, S. Kim, and A. Poon, "Implantable biomedical devices: Wireless powering and communication," IEEE Commun. Mag., vol. 50, no. 4, pp. 152-159, Apr. 2012.

[3] D. Nikolayev, M. Zhadobov, R. Sauleau, and P. Karban, "Antennas for ingestible capsule telemetry," in Advances in Body-Centric Wireless Communication: Applications and State-of-the-Art, London, UK: IET, 2016, pp. 143-186.

[4] A. K. Skrivervik, "Implantable antennas: The challenge of efficiency," in Proc. 7th Eur. Conf. on Antennas and Propagation (EuCAP 2013), Gothenburg, Sweden, 2013, pp. 3627-3631.

[5] D. Nikolayev, M. Zhadobov, P. Karban, and R. Sauleau, "Conformal antennas for miniature in-body devices: The quest to improve radiation performance," URSI Rad. Sci. Bull., vol. 363, pp. 52-64, Dec. 2017.

[6] S. Benaissa et al., "Experimental characterization of in-to-out-body path loss at $433 \mathrm{MHz}$ in dairy cows," Electron. Lett., vol. 55, no. 7, pp. 422 424, Apr. 2019.

[7] J. Faerber et al., "In vivo characterization of a wireless telemetry module for a capsule endoscopy system utilizing a conformal antenna," IEEE Trans. Biomed. Circuits Syst., vol. 12, no. 1, pp. 95-105, Feb. 2017.

[8] S. Perez-Simbor, C. Andreu, C. Garcia-Pardo, M. Frasson, and N. Cardona, "UWB path loss models for ingestible devices," IEEE Trans. Antennas Propag., Jan. 2019.

[9] S. Bakogianni and S. Koulouridis, "On the design of miniature MedRadio implantable antennas," IEEE Trans. Antennas Propag., vol. 65, no. 7, pp. 3447-3455, Jul. 2017.

[10] Z. Jiang et al., "Wideband loop antenna with split ring resonators for wireless medical telemetry," IEEE Antenn. Wireless Propag. Lett., vol. 18, no. 7, pp. 1415-1419, Jul. 2019.

[11] W. Lei and Y. Guo, "Design of a dual-polarized wideband conformal loop antenna for capsule endoscopy systems," IEEE Trans. Antennas Propag., vol. 66, no. 11, pp. 5706-5715, Nov. 2018.

[12] F. Merli et al., "Design, realization and measurements of a miniature antenna for implantable wireless communication systems," IEEE Trans. Antennas Propag., vol. 59, no. 10, pp. 3544-3555, Oct. 2011.

[13] M. S. Miah et al., "Antenna system design for improved wireless capsule endoscope links at $433 \mathrm{MHz}$," IEEE Trans. Antennas Propag., vol. 67, no. 4, pp. 2687-2699, Apr. 2019.

[14] D. Nikolayev, M. Zhadobov, L. Le Coq, P. Karban, and R. Sauleau, "Robust ultra-miniature capsule antenna for ingestible and implantable applications," IEEE Trans. Antennas Propag., vol. 65, no. 11, pp. 61076119, Nov. 2017.

[15] Y. Peng , K. Saito, and K. Ito, "Antenna design for impulse-radiobased wireless capsule endoscope communication systems," IEEE Trans. Antennas Propag., vol. 66, no. 10, pp. 5031-5042, Oct. 2018.

[16] J. Wang et al., "An implantable and conformal antenna for wireless capsule endoscopy," IEEE Antenn. Wireless Propag. Lett., vol. 17, no. 7, pp. 1153-1157, Jul. 2018.

[17] Z. Duan, L. J. Xu, S. Gao, and W. Geyi, "Integrated design of wideband omnidirectional antenna and electronic components for wireless capsule endoscopy systems," IEEE Access, vol. 6, pp. 29626-29636, May 2018.

[18] Y. Feng, Y. Li, L. Li, B. Ma, H. Hao, and L. Li, "Tissuedependent co-matching method for dual-mode antenna in implantable neurostimulators," IEEE Trans. Antennas Propag., to be published. doi: 10.1109/TAP.2019.2916736.

[19] D. Nikolayev, M. Zhadobov, and R. Sauleau, "Immune-to-detuning wireless in-body platform for versatile biotelemetry applications," IEEE Trans. Biomed. Circuits Syst., vol. 13, no. 2, Jan. 2019.

[20] M. K. Magill, G. A. Conway, and W. G. Scanlon, "Tissue-independent implantable antenna for in-body communications at $2.36-2.5 \mathrm{GHz}$," IEEE Trans. Antennas Propag., vol. 65, no. 9, pp. 4406-4417, Sep. 2017.
[21] Z. Bao, Y. X. Guo, and R. Mittra, "An ultrawideband conformal capsule antenna with stable impedance matching," IEEE Trans. Antennas Propag., vol. 65, no. 10, pp. 5086-5094, Oct. 2017.

[22] Z. Bao, Y.-X. Guo, and R. Mittra, "Conformal capsule antenna with reconfigurable radiation pattern for robust communications," IEEE Trans. Antennas Propag., vol. 66, no. 7, pp. 3354-3365, Apr. 2018.

[23] D. Nikolayev, M. Zhadobov, and R. Sauleau, "Impact of tissue electromagnetic properties on radiation performance of in-body antennas," IEEE Antenn. Wireless Propag. Lett., vol. 17, no. 8, pp. 1440-1444, Aug. 2018.

[24] T. Dissanayake, K. P. Esselle, and M. R. Yuce, "Dielectric loaded impedance matching for wideband implanted antennas," IEEE Trans. Microw. Theory Techn., vol. 57, no. 10, pp. 2480-2487, Oct. 2009.

[25] D. Nikolayev, W. Joseph, M. Zhadobov, R. Sauleau, and L. Martens, "Optimal radiation of body-implanted capsules," Phys. Rev. Lett., vol. 122, no. 10, p. 108101, Mar. 2019.

[26] F. Merli, B. Fuchs, J. R. Mosig, and A. K. Skrivervik, "The effect of insulating layers on the performance of implanted antennas," IEEE Trans. Antennas Propag., vol. 59, no. 1, pp. 21-31, Jan. 2011.

[27] A. K. Skrivervik, M. Bosiljevac, and Z. Sipus, "Fundamental limits for implanted antennas: Maximum power density reaching free space," IEEE Trans. Antennas Propag., vol. 67, no. 8, pp. 4978-4988, Aug. 2019.

[28] D. Nikolayev, M. Zhadobov, P. Karban, and R. Sauleau, "Electromagnetic radiation efficiency of body-implanted devices," Phys. Rev. Applied, vol. 9, no. 2, p. 024033, Feb. 2018.

[29] S. Gabriel, R. W. Lau, and C. Gabriel, "The dielectric properties of biological tissues: II. Measurements in the frequency range $10 \mathrm{~Hz}$ to 20 GHz," Phys. Med. Biol., vol. 41, pp. 2251-2269, Nov. 1996.

[30] A. Kiourti, K. A. Psathas, and K. S. Nikita, "Implantable and ingestible medical devices with wireless telemetry functionalities: A review of current status and challenges," Bioelectromagnetics, vol. 35, no. 1, pp. 1-15, Jan. 2014.

[31] Y. H. Jung et al., "A compact parylene-coated WLAN flexible antenna for implantable electronics," IEEE Antenn. Wireless Propag. Lett., vol. 15 , pp. 1382-1385, 2016.

[32] Z. Duan, Y.-X. Guo, M. Je, and D.-L. Kwong, "Design and in vitro test of a differentially fed dual-band implantable antenna operating at MICS and ISM bands," IEEE Trans. Antennas Propag., vol. 62, no. 5, pp. 2430-2439, May 2014.

[33] K.-L. Wong, Design of Nonplanar Microstrip Antennas and Transmission Lines, 1st ed. New York, NY: Wiley-Interscience, 1999.

[34] S. Koulouridis, G. Kiziltas, Y. Zhou, D. J. Hansford, and J. L. Volakis, "Polymer-Ceramic Composites for Microwave Applications: Fabrication and Performance Assessment," IEEE Trans. Microw. Theory Techn., vol. 54, no. 12, pp. 4202-4208, Dec. 2006.

[35] S. Lee et al., "A study on dielectric constants of epoxy/SrTiO3 composite for embedded capacitor films (ECFs)," IEEE Trans. Adv. Packag., vol. 30, no. 3, pp. 428-433, Aug. 2007.

[36] J. Anguera, L. Boada, C. Puente, C. Borja, and J. Soler, "Stacked Hshaped microstrip patch antenna," IEEE Trans. Antennas Propag., vol. 52, no. 4, pp. 983-993, Apr. 2004.

[37] C. A. Balanis, Antenna Theory: Analysis and Design, 4th ed. Hoboken, NJ: John Wiley \& Sons, 2016.

[38] D. Nikolayev, Z. Kubík, P. Karban, and J. Skála, "Impedance analysis of transmission line cells for EMC applications using Agros2D," Appl. Math. Comput., vol. 289, pp. 381-387, Oct. 2016.

[39] Computer Simulation Technology AG, CST Microwave Studio, Feb. 20, 2018. [Online]. Available: http://www.cst.com

[40] D. Nikolayev, M. Zhadobov, P. Karban, and R. Sauleau, " $434 \mathrm{MHz}$ ISM band antenna for in-body biotelemetry capsules," in Proc. 11th Eur. Conf. on Antennas and Propagation (EuCAP 2017), Paris, France, 2017, pp. 1035-1038.

[41] D. Nikolayev, "Modeling and characterization of in-body antennas," in Proc. 17th Int. Conf. Mathematical Methods in Electromagnetic Theory (MMET 2018), Kyiv, Ukraine, 2018, pp. 42-46.

[42] "IEEE Standard for Safety Levels with Respect to Human Exposure to Electric, Magnetic and Electromagnetic Fields, $0 \mathrm{~Hz}$ to $300 \mathrm{GHz}$," IEEE Standard C95.1-2019, 2019.

[43] TCI Ceramics, $K-80$, Feb. 20, 2018. [Online]. Available: http:// www.magneticsgroup.com/m_dielec.htm

[44] C. G. Malmberg and A. A. Maryott, "Dielectric constants of aqueous solutions of dextrose and sucrose," J. Res. Natl. Bur. Stand., vol. 45, no. 4, pp. 299-303, 1950.

[45] Schmid \& Partner Engineering AG, Dielectric Assessment Kit, Jul. 02, 2018. [Online]. Available: http://www.speag.com/products/dak/ dielectric-measurements/ 
[46] enprobe GmbH, LFA-3 analog fiber optic link, Jul. 02, 2018. [Online]. Available: http://www.enprobe.de/products_FO-Links.htm
[47] ETS-Lindgren, 3164-06 Quad-Ridged Horn, Feb. 20, 2018. [Online]. Available: http://www.ets-lindgren.com/3164-06 